\title{
Deepening the Understanding of Mathematics with Geometric Intuition
}

\author{
Qin Lin, Yumei Chen* \\ China West Normal University, Nanchong 637000, Sichuan Province, China \\ *Corresponding author: Yumei Chen, 296079800@qq.com
}

\begin{abstract}
Geometric intuition is one of the core concepts introduced by the new mathematical curriculum standards. It aims to use intuition and intuitive materials to deepen the understanding of mathematics in mathematical cognition activities. It does not only play a role in the learning of "graphics and geometry,' but its' irreplaceable role also involves the whole process of mathematics education. Therefore, if teachers can skillfully use geometric intuition in the teaching process, classroom efficiency will be greatly improved.
\end{abstract}

Keywords: Geometric intuition; Mathematics education

Publication date: June 2021; Online publication: June 30, 2021

\section{Introduction}

The famous mathematician, Lizhi Xu stated that "Intuition is the direct perception and understanding of the relationship between things by means of experience, observation, testing or analogy while geometric intuition is the image of seeing or thinking of geometric figures. Relationships produce intuitive perceptions of quantitative relationships." ${ }^{[1]}$ Meaning to say, intuition helps to establish the relationship between foreign object experiences and self-perception, simplify complex mathematical problems, explore ideas for solving problems, and predict results. Therefore, if teachers can apply geometric intuition to their teaching contents, they would be able to achieve twice the results with only half the effort.

\section{Geometrically intuitive concrete manifestations}

Combining the materials as well as the actual teaching practices of mathematics in elementary and middle schools, the concrete expressions of geometric intuition can be divided into three types which are object intuition, intuitive diagrams, and graphical intuition.

Object intuition directs students to observe reality in relation to the research object and use it to intuitively perceive the characteristics of the image or quantitative relationship of the research object. ${ }^{[2]}$ For example, in the learning of "three different views" in junior high school mathematics, in order to accurately distinguish the three different views of simple objects and understand the relationship of their position and size in each view, teachers would ask students to observe the objects around them such as chalk boxes, stationery boxes, water cups, etc. In this way, students would have a deep impression regarding the subject about three different views.

Intuitive diagrams refer to the use of brief symbols, condensed text, lines (arrows), etc. to form a unique diagram to intuitively express the internal relationship of the research object. For example, car A and B are parked at point $\mathrm{A}$ and $\mathrm{B}$ respectively in which they are 240 kilometers apart. 


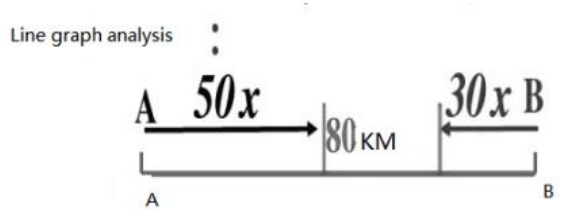

Figure 1.

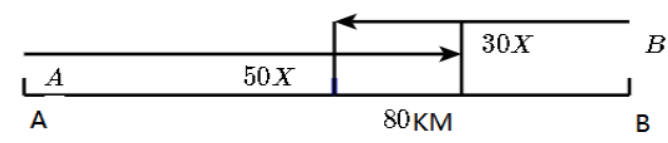

Figure 2.

It is known that car A and car B can travel at 50 kilometers per hour and 30 kilometers per hour, respectively. If the two cars start moving towards each other at the same time, how long will it take for car B to be 80 kilometers apart from car A? In the above representation, students would abstract the realistic prototype to a certain degree and use symbolic diagrams as shown in the figures above to clearly and concisely demonstrate the relationship between the direction of movement of the objects and the magnitude of the displacement in order to solve the problem.

Graphical intuition refers to an intuitive way of using clear geometrical figures to help students understand abstract mathematical information and solve complex problems. With the help of the intuitiveness of graphics, students would understand abstract algebra from calculating the area of geometric figures as shown in Figure 3.

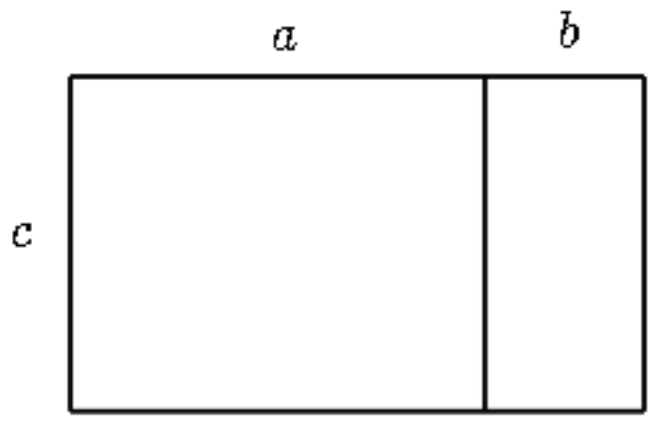

Figure 3.

\section{Specific application of geometric intuition in teaching}

Geometry intuition plays an important role in all aspects of mathematics learning with the intuitive advantage of objects, diagrams, and graphics.

\subsection{Using geometric intuition to understand mathematical concepts}

Klein proposed that the intuition of mathematics is the direct grasp of concepts and proofs. In the process of mathematics learning, the grasp of mathematical abstract concepts and proofs happens to be one of the difficulties that students often encounter. ${ }^{[3]}$ Students may remember the conceptual knowledge but they cannot apply the relevant conceptual knowledge to solve problems. The fundamental reason for this phenomenon is that students have not thoroughly understood abstract concepts and they are still stuck at the mechanical memory state. Therefore, teachers should be proficient in using geometric intuition to vividly present abstract mathematical knowledge in the form of objects, diagrams, or graphics so that 
students would not only deepen their understanding in regard to abstract concepts but also achieve the goal of dynamic learning and application.

Using the "opposite number" teaching as an example, teachers should guide their students to think about three groups of numbers on the number axis by observing the positional relationship between +1 and $-1,+2.5$ and -2.5 , as well as +5 and -5 as shown in Figure 4. Then, students would need to determine the characteristics of the groups of numbers.

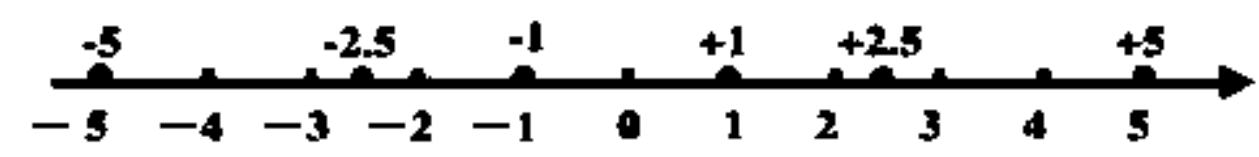

Figure 4.

Students would then be able to find that the above three sets of numbers have the characteristics of different signs but with consistent values. They would also be able to observe and analyze the positional relationship of the points on the number axis and intuitively recognize that the three sets of numbers are located on opposite sides in regard to the origin while their distances from the origin are equal. Therefore, the use of the number line does not only help students to review their existing mathematical knowledge but also intuitively displays the characteristics of two numbers that are opposite to each other which then deepens students' understanding of the concept of opposites.

\subsection{Proving theorems with geometric intuition}

As an important aspect in mathematics teaching, the proving of theorems is also regarded as a major activity. The process of students proving theorems is likely to be used in the process of solving practical problems in the future. Therefore, in the teaching of theorems, if teachers can integrate materials from textbooks with reality and effectively use geometric intuition to prove theorems, it will be helpful to the understanding and memorization of knowledge.

Using "Pythagorean Theorem" as an example, teachers should implore students to try to form a large square comprising of congruent triangles with four right-angle sides, $a$ and $b$, hypotenuse $c$ and a square with sides, c as shown in Figure 5.

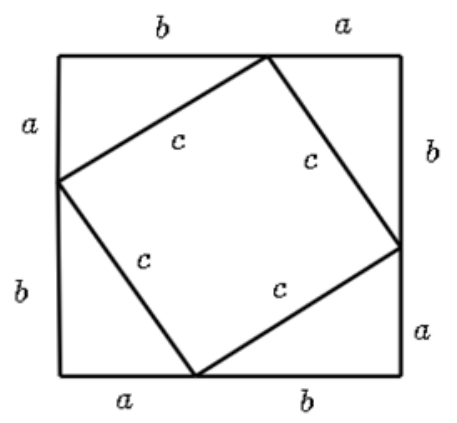

Figure 5 .

In the hands-on process, it stimulates students' interests in learning, cultivate the awareness of cooperation, communication, and the spirit of exploration, as well as deepen their mathematical knowledge. 


\subsection{Using geometric intuition to prove mathematical formulas}

From elementary school to middle school, there are countless mathematical formulas that students have learned and memorized. However, in teaching, it is not difficult that some formulas are likely to be psychologically rejected due to their complicated processes. Hence, resulting in the "only knowing what is happening and not knowing why" phenomenon and ultimately, students are unable to flexibly use the formula learned in the process to solve real problems. Therefore, learning the method to prove formulas is not only helpful in understanding knowledge but it also would exercise students' mathematical thinking. If teachers could skillfully use geometric intuition to reduce complexity, it would be of great help to enhance the effectiveness of lessons and broaden the students' horizons.

The "perfect square formula" is used as an example. As the main multiplication formula, it provides an important basis for students' future calculations. Therefore, the focus of teaching the formula is not only to allow students to learn its application in simple calculations but also for them to understand the essence of formula as well as to experience the process of formula discovery and derivation. In teaching, teachers should design interesting practical activities and encourage students to cooperate in groups. Students should then try to combine two square cards with sides $a$ 、 $b$, and a rectangular card with length of a and width of $b(a<b)$ into two squares with the length of the sides is enlarged by $b$, and the length of the sides is reduced by b. As shown in Figure 6:
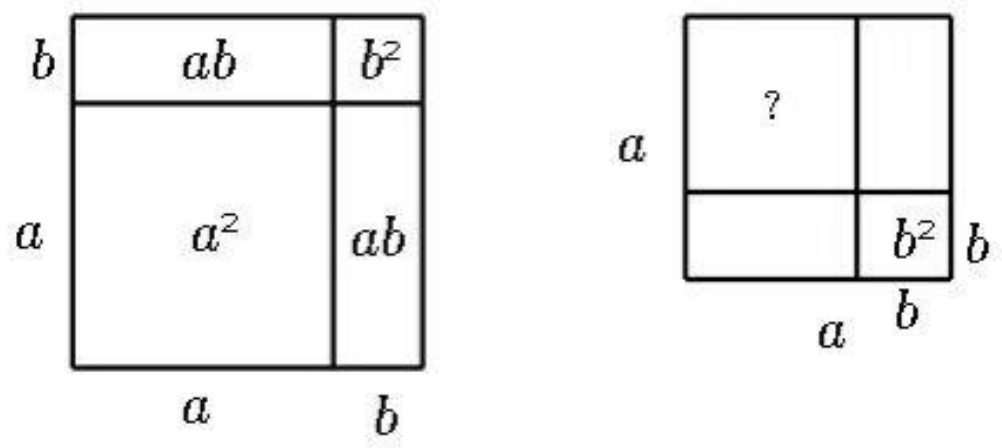

Figure 6.

With the help of the above vivid graphics, students would be able to intuitively perceive the discovery and derivation process of the complete square formula and cultivate their reasoning ability and creative thinking.

\subsection{Solving mathematical problems with geometric intuition}

"Questions begin with questions and doubts arise from thinking." Mathematics learning is a complex thinking process and the mathematical problems contained in it can be seen as various obstacles encountered in the thinking process. If these obstacles are resolved smoothly, it will undoubtedly be of great help to the advancement of mathematical thinking. In the process of solving mathematical problems, geometric intuition provides a new method to allow abstract mathematical problems to be more intuitive and explicit. Thus, helping students to solve mathematical problems better and understand mathematical knowledge. For example:

$$
a, b, c, A, B, C \in R^{+} \text {and } a+A=b+B=c+C=k \quad \text { verify } a B+b C+c A<k^{2} \text {. }
$$


If students derive the proof from the algebraic point of view, the process will be cumbersome and difficult to understand. However, if the algebraic information can be transformed into geometric information to be regarded as geometric models formed by the edges of certain figures, then the problem mentioned above will eventually be solved as shown in Figure 7.

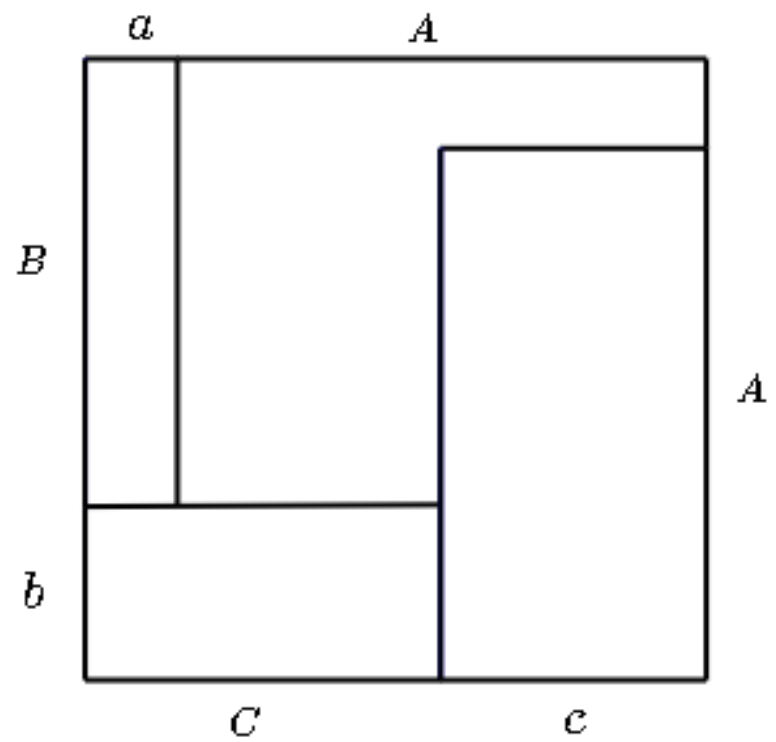

Figure 7.

By transforming an algebraic problem into a geometric model, the originally abstract mathematical problem becomes intuitive. It not only helps students solve actual math problems but is also a breakthrough of the obstacles in terms of students' thinking; hence, allowing them to fully experience the charm of geometric intuition.

All in all, in mathematics teaching, teachers should combine teaching materials, make full use of their own educational wit, and use appropriate objects, diagrams, or graphics to help students understand abstract mathematical knowledge and simplify complex problem-solving steps. In this way, students would be able to experience the joy of mathematics learning in the process of exploration and discovery while cultivating good mathematical thinking and experiencing the scientific worldview and methodology.

\section{Disclosure statement}

The author declares no conflict of interest.

\section{References}

[1] Xu L, 2000, Talk about some of my experience in mathematics. Mathematics Bulletin, (5).

[2] Wang, Q, 2019, The connotation, classification, and teaching essentials of geometric intuition. Primary and secondary school teacher training, (6): 60-4.

[3] Shi Y, 2021, Use geometric intuition to highlight classroom effectiveness. Reading and Writing, (3): $0130,0272$. 